\title{
Design and Error Compensation Performance of a Precision Micro-Drive Rotary System
}

\author{
Manzhi Yang $\mathbb{D}^{1},{ }^{1}$ Gang Jing $\mathbb{D}^{1},{ }^{1}$ Zhenyang Lv, ${ }^{1}$ Wei Guo, ${ }^{1}$ Yumei Huang, ${ }^{2}$ Kaiyang Wei $\mathbb{D}^{1},{ }^{1}$ \\ Linyue Li $\mathbb{D},{ }^{1}$ Bin Feng, ${ }^{1}$ Hongyu Ge, ${ }^{1}$ and Shuaitian $\mathrm{Li}^{1}$ \\ ${ }^{1}$ College of Mechanical Engineering, Xi'an University of Science and Technology, No. 58 Yanta Middle Road, Xi'an, \\ Shaanxi 710054, China \\ ${ }^{2}$ School of Mechanical and Precision Instrumental Engineering, Xi'an University of Technology, No. 8 Jinhua South Road, Xi'an, \\ Shaanxi 710048, China
}

Correspondence should be addressed to Manzhi Yang; xkdymz@xust.edu.cn

Received 23 April 2021; Accepted 17 June 2021; Published 20 July 2021

Academic Editor: Shanglei Jiang

Copyright (C) 2021 Manzhi Yang et al. This is an open access article distributed under the Creative Commons Attribution License, which permits unrestricted use, distribution, and reproduction in any medium, provided the original work is properly cited.

In order to obtain motion with large travel and high precision, the micro-drive system is used to compensate for the motion error of the macro-drive system in the macro/micro dual-drive system. The research on the micro-drive rotary system lags behind the micro-drive linear system, so it is of great significance to study the designing and error compensation performance of a precision micro-drive rotary system. In this paper, a precision micro-drive rotary system is designed, the error compensation scheme of the system is proposed, and the system feasibility in design and error compensation is tested by FEM simulation analysis and performance experiments. Firstly, a precision micro-drive rotary system is designed to provide high-precision rotary motion, which consists of a micro rotary mechanism and PZT. In the system, the micro rotary mechanism is developed based on the compound motion principle of flexure hinge, which can accurately transform an input of linear motion into an output of rotary motion according to a certain relationship. Secondly, for finishing the error compensation scheme of the system, the maximum compensation modifier $\theta_{\max }^{\prime}$ is proposed based on the analysis of error compensation equations of point-to-point motion and continuous motion. Finally, in order to facilitate the use of engineering, the driven voltage equation of error compensation is derived by the error compensation performance experiment. The simulation and experiment results indicate that both the design and error-compensation-range of the system satisfy the needs of practical application.

\section{Introduction}

Mechanical equipment manufacturing is the key of the manufacturing industry. Because the precision requirements of mechanical systems in the modern industry continually improve, the machining precision and motion precision requirements of equipment manufacturing mechanical systems continually increase [1-3]. At present, precision and ultra-precision processing technologies are important realization methods of mechanical equipment precision manufacturing [4-10]. The precision feed technology is an important realization method of ultra-precision processing technology [11-13]. In addition, the macro/micro dual-drive system solves the contradiction between large-motion travel and high motion precision, which can provide motion with large travel and high precision. The macro/micro dual-drive system has been widely used in high-technology fields such as the advanced war industry, biological medicine, and precision electronics [14-19].

In the macro/micro dual-drive system, as macro-actuators (i.e., direct drive motor) have their own defects such as vibration and motion pulse error, the macro-drive system can only provide large-travel displacement, but it cannot provide motion in ultrahigh precision (i.e., nanometer level or sub-arc-second level) even after error compensation. However, a micro-drive system can reach ultra-high precision because of ultra-precision drive by micro-actuators and the good transmission performance of the micro 
mechanism. The micro-drive system can compensate for the motion error of the macro-drive system with a higher precision level, so the macro/micro dual-drive system can provide motion with large travel and high precision [20-23]. By providing accurate motion based on the compound motion principle of the flexure hinge, the micro-mechanism is the core part of the micro-drive system [24-27]. Therefore, it is necessary to study the performance of the micro-drive system.

In recent years, many scholars studied the micro-drive system, and they mainly focused on certain research areas, such as mechanical structure design, structure optimization, dynamic analysis, and practical application. A precision MEMS (Micro-electromechanical System) mechanism with six degrees of freedoms was designed, which can be applied to electron transport micro-scopes with the size of $10 \mu \mathrm{m} \times 20 \mu \mathrm{m} \times 0.2 \mu \mathrm{m}$; in addition, the mechanism has realized precision rotary motion in three-dimensional space [28]. A bridge micro-mechanism with three degrees of freedoms was developed, and the $Z$ direction of the mechanism has high stiffness and stability, which can resist large external thrust. The structure of the mechanism was optimized by the sequential quadratic rule, and the experiment results show that the optimization method is very effective [29]. A flexible micro-mechanism with two-directional rotary motion driven by Giant Magnetostrictive Actuator was proposed, and the dynamic model of mechanism parameters was given based on the dynamic model establishment [30]. A new chip-level comb-drive magnifying micro-drive platform with three degrees of freedom $\left(X_{-}, Y_{-}\right.$, and $Z$-directions) was designed, and the platform can achieve large displacements of $25.2 \mu \mathrm{m}$ in $x$-direction, 20.4 $\mu \mathrm{m}$ in $y$-direction, and $58.5 \mu \mathrm{m}$ in $z$-direction [31]. A new compliant mechanism constituted by flexure hinges with two-straight circular and multinotched was mentioned, whose structure optimization was accomplished using the topological method; the mechanism performances were analyzed by the finite element method, including the stiffness, rotary precision, and horizontal stress, and the analysis results show that the mechanism performances are excellent [32]. A fully compliant, potentially monolithic, power transmission mechanism, which can rectify a large lateral offset between two parallel rotational axes, was developed; the internal stiffness is removed by static balancing and results in a statically balanced compliant transmission mechanism, that is, with zero actuation stiffness [33].

At present, the research on the micro-drive system mainly concentrates on linear motion, and many studies have been used in production [34-36]. However, research on the micro-drive rotary system is rare, and the positional accuracy is concentrated on the arc-second (") level [37, 38]. Therefore, a new micro-mechanism is badly in need of transforming the linear motion into rotary motion with high-precision, and then we can obtain high-precision rotary motion based on the ultra-high precision of linear motion. In this paper, a micro rotary system is designed which can solve the low-precision problem of the rotary system. In this system, a new micro rotary mechanism is developed based on the compound motion principle of flexure hinge, and an ultra-high-precision PZT as microactuators to drive the micro-mechanism.

Additionally, the error compensation performance of the micro-drive rotary system can directly affect the motion precision of the macro/micro dual-drive rotary system. Therefore, it is significant to design an error compensation analysis for the micro-drive rotary system.

A precision micro rotary system is designed to obtain the rotary motion with high precision. The precision micro rotary system could convert the linear displacement into the rotary displacement accurately, thus solving the problem of low precision of rotary motion and proposing the error compensation scheme of the system.

The driven voltage equation of error compensation is derived by the error compensation performance experiment. The simulation and experiment results show that both the design and error compensation performance of the system satisfy the needs of practical application. This study has reference significance to research on design and error compensation performance of the micro-drive rotary system.

The rest of this paper is organized as follows: Section 2 introduces the design of the micro-drive rotary system. The error compensation scheme of the system is given in Section 3. In Section 4, strength and modal analysis of the system by FEM simulation are finished. Section 5 carries out modal and error compensation performance experiments and analyzes results. The conclusions are drawn in Section 6 .

\section{Design of the Micro-Drive Rotary System}

At the macro/micro dual-drive system, the macro-drive system provides the motion with large travel, and the microdrive system compensates the position error of the macrodrive system; therefore, the macro/micro dual-drive system has high-precision and large-travel motion. To develop a macro/micro dual-drive rotary system with high precision and large travel, a precision micro-drive rotary system is designed, and the system uses the PZT as a micro-actuator to drive the micro rotary mechanism to obtain high-precision rotary motion. The micro-drive rotary system is shown in Figure 1.

2.1. The Micro Rotary Mechanism. When a flexible material is stressed, the flexure hinge will produce minor deformation, which can realize the transmission motion and guiding motion; this principle is the compound motion principle of the flexure hinge. The transmission motion amplifies or reduces an input motion with a certain ratio, and transmission motion can also sometimes transform motion between linear and rotary with a certain relationship. The guiding motion guides the direction of motion by flexure hinges, which can ensure that there is a displacement of motion only in motion direction, and there is no nonmotion direction displacement during motion.

In this paper, a micro rotary mechanism with singleDOF symmetric drive and guiding and transformation is designed based on the compound motion principle of 


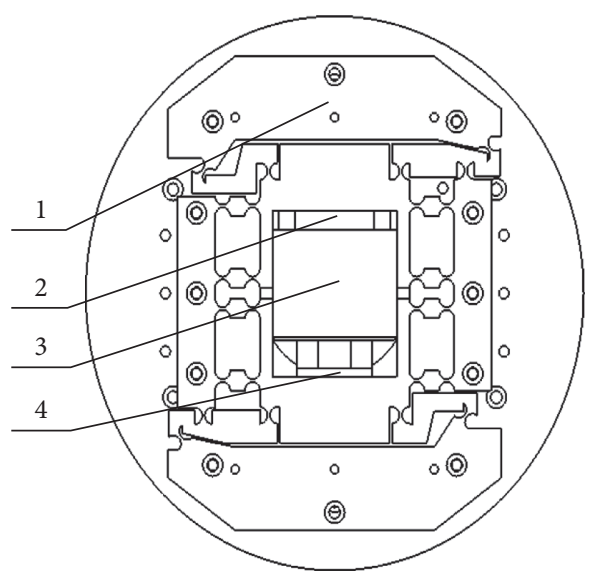

(a)

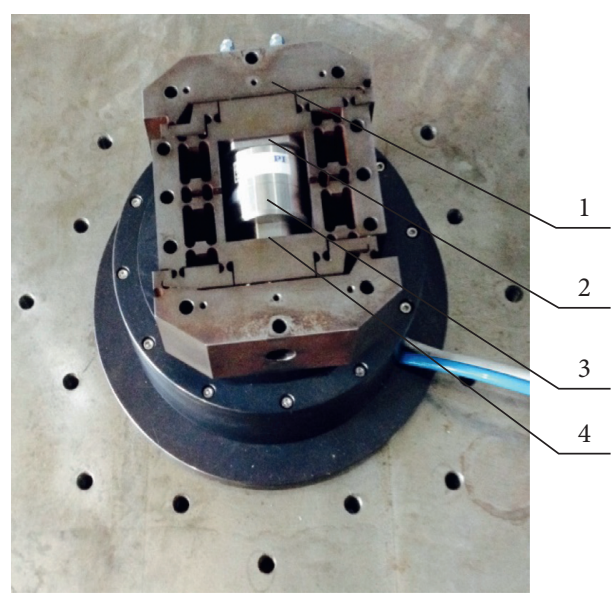

(b)

Figure 1: The micro-drive rotary system. (a) Schematic diagram of the micro-drive rotary system. (b) Picture of the micro-drive rotary system. (1) The micro rotary mechanism. (2) Gasket. (3) PZT (piezoelectric actuator). (4) Fixing sleeve.

flexure hinge, which can accurately transform the input of linear motion into an output of rotary motion according to a certain relationship (i.e., realize the accurate transformation of $\Delta u$ to $\Delta \theta$ ). The micro rotary mechanism is shown in Figure 2, and the stereogram of the mechanism is shown in Figure 2(a). The micro rotary mechanism consists of four parts: $a, b, c$, and $d$. Part $a$ consists of the connecting element between macro-drive and micro-drive, which is the element that connects the motion of the macro-drive with the motion of the micro-drive. Part $b$ is the input motion element of the micro-drive, which brings the input linear motion $(\Delta u)$ of PZT to the micro rotary mechanism. Part $c$ is the flexure hinge element, which transforms and guides. Part $d$ is the output motion element, which delivers the output rotary motion $(\Delta \theta)$ of the micro rotary mechanism to the rotary workbench.

The structure and working principle of the micro rotary mechanism as shown in Figure 2(b); there are 26 flexure hinge elements in the $x-y$ plane with symmetrical distribution about center point 0 , and the 26 flexure hinge elements having the same size are numbered as shown in Figure 2(b). Because the mechanism has a symmetrical structure, it is feasible only to study the top half of the mechanism when we analyze its working condition. The symmetrical structure parts of $a$ contain $a_{1}$ (at the left half parts of the mechanism) and $a_{2}$ (at the right half parts of the mechanism). Similarly, $b$ contains $b_{1}$ (at the top half of the mechanism) and $b_{2}$ (at the bottom half of the mechanism), and $d$ contains $d_{1}$ (at the top half of the mechanism) and $d_{2}$ (at the bottom half of the mechanism). The pivots of flexure hinges $2,6,8,11$, and 13 are connected with $a . a_{1}$ and $a_{2}$ are each fixed by $3 \mathrm{M} 4$ socket head cap screws (the six screws are simplified for simple construction). The pivots of flexure hinges 3 and 5 are connected with $d_{1}$, and $d_{1}$ and $d_{2}$ are each connected with the rotary workbench by $3 \mathrm{M} 4$ socket head cap screws (the six screws are simplified for simple construction). The pivots of flexure hinges $1,4,7,9,10$, and 12 are connected with $b_{1}$. The connecting rod 123 (which consists of points 1,2 , and 3; other connecting rods are similarly defined) completes the transform from linear motion to rotary motion. Flexure hinges 6-13 complete the guiding for the mechanism, and the mechanism will have no non-motion direction displacement (the $y$-axis is the motion direction). Flexure hinges 4 and 5 are used to balance the $x$ direction force and moment because flexure hinge 1 moves around the pivot of flexure hinge 2 , while the mechanism is working.

We establish the rectangular coordinate system as shown in Figure 2(b). When PZT has a micro-drive displacement $\Delta u$ along the forward direction of the $y$ axle, part $b_{1}$ has displacement $\Delta u$. Simultaneously, the pivots of flexure hinges 1 and 4 have $x$-axle displacement $\Delta u$, and after the motion, the pivot of flexure hinges 1 and 4 will move to $1^{\prime}$ and $4^{\prime}$. The pivot of flexure hinges 3 and 5 will move to $3^{\prime}$ and $5^{\prime}$; because they are constrained by part $d_{1}$, the distance between $3^{\prime}$ and center point 0 is equal to the distance from 3 to center point 0 (distance $r$ is constant). As constrained by parts $b_{1}$ and $d_{1}$, at connecting rod $123, l_{12}$ (length from point 1 to point 2 ) will shorten, $l_{23}$ (length from point 2 to point 3 ) will elongate, $l_{13}$ (length from point 1 to point 3$)=l_{1^{\prime} 3^{\prime}}$ (length from point $1^{\prime}$ to point $3^{\prime}$ ), and the location of point $3^{\prime}$ can be calculated. The included angle is between $L_{03}$ (the connect line from point 0 to point 3 ) and $L_{03^{\prime}}$ (the connect line from point 0 to point $3^{\prime}$ ), and the angle is the output rotary motion displacement $(\Delta \theta)$.

Additionally, because the mechanism has a symmetrical structure, the bottom half of the mechanism has the identical output rotary motion displacement $(\Delta \theta)$ driven by PZT with an input linear motion displacement $(\Delta u)$. This motion process of the mechanism will accurately transform the input linear motion $(\Delta u)$ to the output rotary motion $(\Delta \theta)$. The PZT will not bear the force or torque with non-motion 


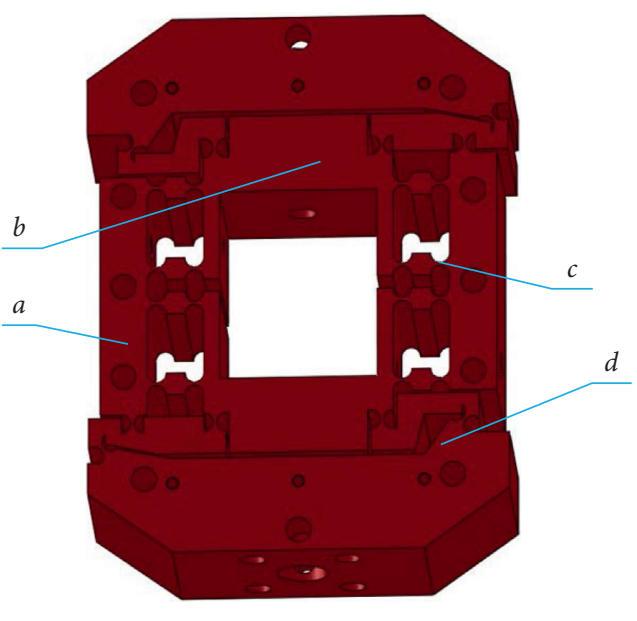

(a)

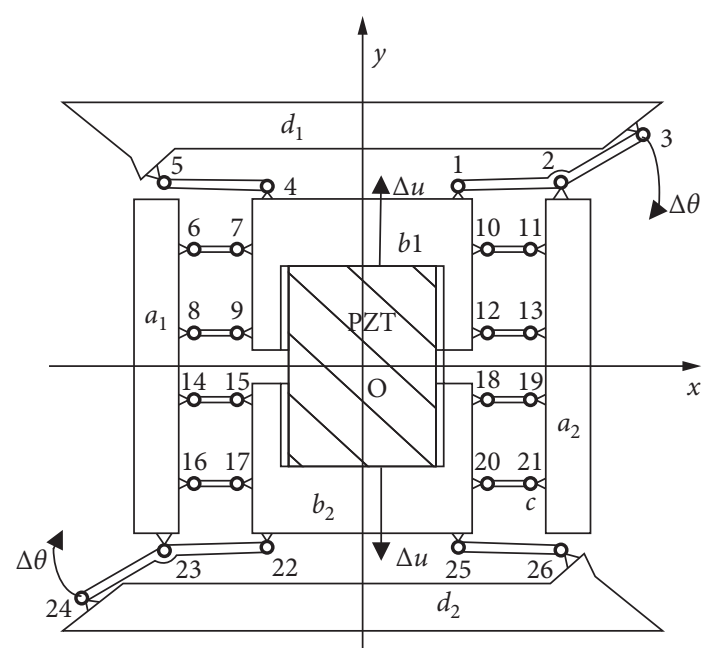

(b)

FIgURE 2: The micro rotary mechanism. (a) The stereogram of the mechanism. (b) Working principle of the micro rotary mechanism.

direction, and the mechanism does not have non-motion direction displacement, which can ensure the precision and stationary of the mechanism motion.

2.2. Micro Actuators. Two common micro-actuators are PZT (piezoelectric actuator) and GMA (giant magnetostrictive actuator). The motion accuracy of PZT is higher than that of GMA.

PZT is one kind of the micro-drivers, which works on the principle inverse piezoelectric effect of piezoelectric materials, and PZT can produce displacement of several micrometers to tens of micrometers. Because of advantages such as high precision, rapid response, small size, and largeoutput-force, PZT has been used widely in fields such as the advanced war industry, biological medicine, precision electronics, intelligent robot, and precision machinery. According to the parameter demand of this system, such as the size of the driver, driving force, and driving displacement, the model of the high-precision PZT is P-235.1S of PI Company; the PZT is shown in Figure 3. The main parameters of the PZT are shown in Table 1, closed-loop travel is $15 \mu \mathrm{m}$, the closed-loop resolution is $0.3 \mathrm{~nm}$, and the drive frequency is $300 \mathrm{~Hz}$.

The micro rotary mechanism is designed as shown in Section 2.1, and the mechanism is processed by wire-electrode cutting with the spring steel material 60Si2Mn. The micro-drive rotary system is developed as shown in Figure 1, and the fixed part and gasket are designed to connect two ends of the PZT to the micro rotary mechanism.

\section{Error Compensation Scheme of the Micro- Drive Rotary System}

The error compensation scheme of the micro-drive rotary system can directly affect the motion performance of the macro/micro dual-drive system, so it is important to study the scheme.

Two motion methods of the macro/micro dual-drive system are point-to-point motion and continuous motion. In the point-to-point motion, the motions of the macrodrive and micro-drive are out-of-sync: the micro-drive system compensates for the motion error of the macro-drive system after the macro-drive system finishes its motion. In continuous motion, the motions of the macro-drive and micro-drive are synchronous: the micro-drive system compensates for the motion error of the macro-drive system when the macro-drive system is moving.

To calculate the motion error of the macro-drive rotary system, we detect $n$ points in the entire circumference within 360 degrees; the angular position and angular error of the $i$ th point are $x_{i}$ and $\theta_{i}$; and the error of the point is $\left(x_{i}, \theta_{i}\right)$.

Hence, we have the following equation:

$$
x_{i}=(i-1) P_{i}+x_{0},
$$

where $x_{0}$ is the angular value of the initial point, $x_{i}$ is the angular value of the $i$ th detection point, and $P_{i}$ is the angular distance between the $i$ th and $(i-1)$ th detection points.

If the $i$ th detection point is detected $q$ times, the detection error $\theta_{i}$ of the detection points is

$$
\theta_{i}=\frac{1}{q} \sum_{j=1}^{q} \theta_{i j}
$$

where $j$ is the $j$ th time detection in the $q$ times at the $i$ th detection point.

3.1. Point-to-Point Motion. The error compensation equation of the point-to-point motion is

$$
\theta+\theta^{\prime}=0
$$




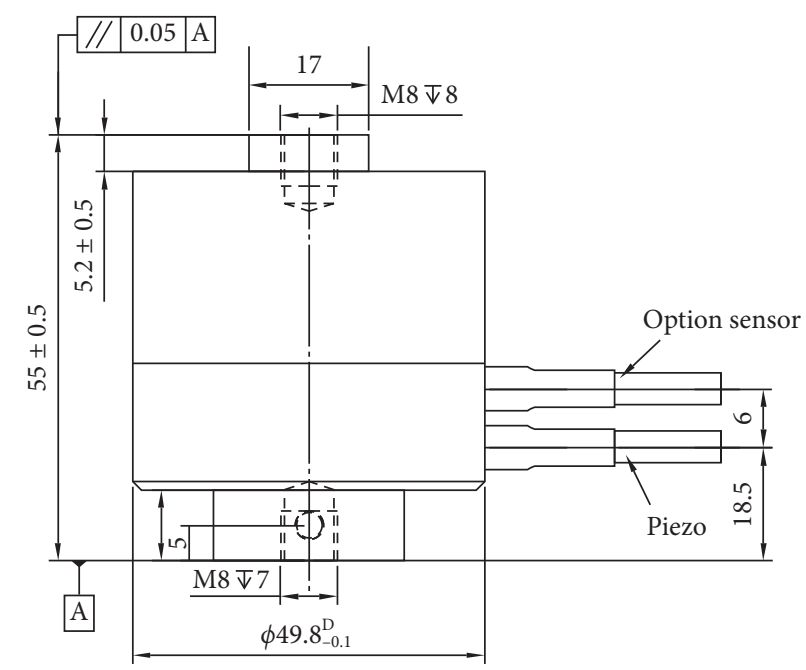

(a)

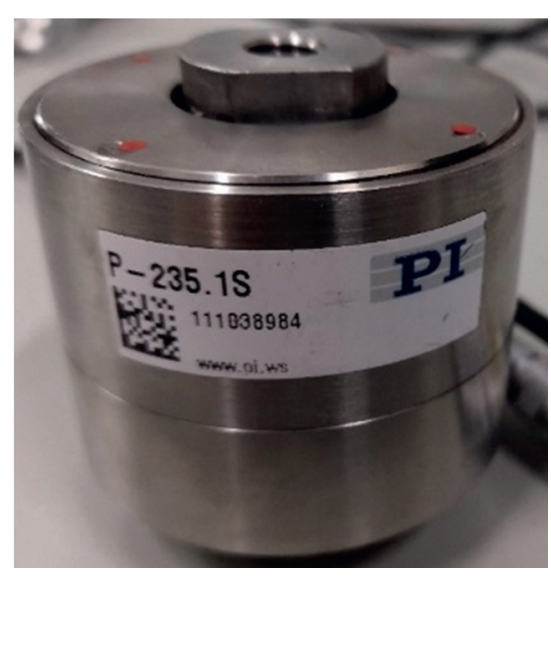

(b)

FIgure 3: PZT model is P235.1S. (a) PZT structure dimension drawing. (b) The picture of the PZT.

TABLE 1: Main parameters of the P235.1S PZT.

\begin{tabular}{lc}
\hline Index & Parameter \\
\hline Length & $55 \mathrm{~mm}$ \\
Cylinder diameter & $49.8 \mathrm{~mm}$ \\
Closed-loop travel & $15 \mu \mathrm{m}$ \\
Resolution & $0.3 \mathrm{~nm}$ \\
Linearity & $0.2 \%$ \\
Static large-signal stiffness & $860 \mathrm{~N} / \mathrm{m}$ \\
Operating voltage & $0-10 \mathrm{~V}$ \\
Maximum push & $30000 \mathrm{~N}$ \\
Maximum pull & $3500 \mathrm{~N}$ \\
Shear force limit & $707 \mathrm{~N}$ \\
Torque limit & $2 \mathrm{Nm}$ \\
Maximum motion frequency & $300 \mathrm{~Hz}$ \\
\hline
\end{tabular}

where $\theta$ and $\theta^{\prime}$ are the error matrix and compensation modifier matrix of each point, $\theta=\left[\begin{array}{llllll}\theta_{0} & \theta_{1} & \ldots & \theta_{i} & \ldots & \theta_{n}\end{array}\right]^{T}$, $\theta^{\prime}=\left[\begin{array}{llllll}-\theta_{0} & -\theta_{1} & \ldots & -\theta_{i} & \ldots & -\theta_{n}\end{array}\right]^{T}$.

The precision evaluation of error compensation for equation (3) is

$$
\sigma^{2}=\frac{S}{n-2}
$$

where $\sigma$ is the residual variance, $s$ is the residual sum of squares, and $n$ is the number of the detection points.

This motion can be compensated by the pitch compensation method and backlash compensation method, and the maximum compensation modifier is

$$
\theta_{\max }^{\prime}=\max \left\{\left|-\theta_{0}\right|\left|-\theta_{1}\right| \ldots\left|-\theta_{i}\right| \ldots\left|-\theta_{n}\right|\right\}
$$

3.2. Continuous Motion. The error compensation equation of continuous motion is a curvilinear equation, and the equation between $x_{i}$ and $\theta_{i}$ is

$$
\theta=f(x)
$$

If $\theta_{i}$, which are the errors of each point $x_{i}$, are known, then the data array $\theta=f(x)\left(x=x_{0}, x_{1}, \ldots, x_{n}\right)$ is known.

If there is an m-order polynomial of $P_{m}(x)=\sum_{k=0}^{m} a_{k} x^{k}$ $(m<n)$, to minimize $R=\sum_{i=0}^{n}\left[f\left(x_{i}\right)-P_{m}\left(x_{i}\right)\right]^{2}, P_{m}\left(x_{i}\right)$ is the fitting polynomial of $(x)$ using the least square method.

Thus, the problem of continuous motion modeling becomes the problem of solving the least square solution about $a_{k}(k=0,1, \ldots, m)$ in the following equation:

$$
\sum_{k=0}^{m} a_{k} x_{i}^{k}=\theta_{i}, \quad(i=0,1, \ldots, n, n>m) .
$$

If $a_{k}$ is a variable, equation (7) can be transformed to the following matrix equation:

$$
\left[\begin{array}{ccccc}
1 & x_{0} & x_{0}^{2} & \ldots & x_{0}^{m} \\
1 & x_{1} & x_{1}^{2} & \ldots & x_{1}^{m} \\
& & & \ldots & \\
1 & x_{n} & x_{n}^{2} & \ldots & x_{n}^{m}
\end{array}\right]\left[\begin{array}{c}
a_{0} \\
a_{1} \\
\vdots \\
a_{m}
\end{array}\right]=\left[\begin{array}{c}
\theta_{0} \\
\theta_{1} \\
\vdots \\
\theta_{n}
\end{array}\right],
$$


which is a hyperpositive definite equation and its corresponding normal equation is

$$
\left[\begin{array}{ccccc}
m+1 & \sum_{i=0}^{n} x_{i} & \sum_{i=0}^{n} x_{i}^{2} & \ldots & \sum_{i=0}^{n} x_{i}^{m} \\
\sum_{i=0}^{n} x_{i} & \sum_{i=0}^{n} x_{i}^{2} & \sum_{i=0}^{n} x_{i}^{3} & \ldots & \sum_{i=0}^{n} x_{i}^{m+1} \\
\ldots & & & \\
\sum_{i=0}^{n} x_{i}^{m} & \sum_{i=0}^{n} x_{i}^{m+1} & \sum_{i=0}^{n} x_{i}^{m+2} & \ldots & \sum_{i=0}^{n} x_{i}^{2 m}
\end{array}\right]\left[\begin{array}{c}
a_{0} \\
a_{1} \\
\vdots \\
a_{m}
\end{array}\right]=\left[\begin{array}{c}
\sum_{i=0}^{n} \theta_{i} \\
\sum_{i=0}^{n} x_{i} \theta_{i} \\
\vdots \\
\sum_{i=0}^{n} x_{i}^{m} \theta_{i}
\end{array}\right] .
$$

Then, $a_{k}$ can be solved using equation (9), and the fitting polynomial of $f(x)$ using the least square method is analyzed as

$$
P_{m}(x)=a_{0}+a_{1} x+a_{2} x^{2}+\cdots+a_{m} x^{m},
$$

which is the error model of continuous motion. The error compensation equation of continuous motion is

$$
\theta^{\prime}(x)=-P_{m}(x)=-a_{0}-a_{1} x-a_{2} x^{2}-\cdots-a_{m} x^{m} .
$$

This motion can be compensated by coupling the error compensation equation (11) with the control program of system motion, and the maximum compensation modifier is

$$
\theta_{\max }^{\prime}=\left|-P_{m}\left(x_{i}\right)\right|_{\max } .
$$

\section{Simulation and Analysis}

4.1. Strength Analysis. In the strength analysis of the microdrive rotary system, because the PZT has sufficiently large stiffness (the PZT can bear a maximum thrust of $3000 \mathrm{~N}$ ), it just needs to check whether the strength of the micro rotary mechanism satisfies the material requirement under the maximum driving displacement by PZT. In this paper, the strength of the micro rotary mechanism is analyzed by the statics module of FEM software.

By importing the 3D model of the micro rotary mechanism into the finite element software, we obtain that the radius and minimum thickness of the flexure hinges are $3 \mathrm{~mm}$ and $1 \mathrm{~mm}$, and the size of the mechanism is $160 \mathrm{~mm} \times 150 \mathrm{~mm} \times 50 \mathrm{~mm}$ (length $\times$ width $\times$ height $). W e$ select the parameters of $60 \mathrm{Si} 2 \mathrm{Mn}$ as the material properties of the mechanism. To conveniently apply loading to the mechanism, we made an imprint face in the mechanism and fit its structure for the inner surface between the mechanism and the PZT. For meshing, we mesh the entire mechanism using the method of free meshing. Then, we refine the grid cell for 52 cylinders of flexure hinges with $0.5 \mathrm{~mm}$ mesh. After meshing, in the meshing model, the number of nodes is 527148; the number is 327190 . During loading, we apply a fixed constraint on the 6 threaded hole cylinders of M4 in part $a$. The maximum displacement of the PZT is $15 \mu \mathrm{m}$, so the $y$-positive-direction and $y$-negative-direction displacements with $7.5 \mu \mathrm{m}$ are loaded at the position of the imprint face, and the preparation of FEM analysis is shown in Figure 4.

The maximum simulation stress of the micro rotary mechanism is shown in Figure 5, and the maximum stress of the mechanism is $30.964 \mathrm{MPa}$.

The allowable stress of the material is

$$
[\sigma]=\frac{\sigma_{s}}{\lambda}
$$

The yield limit $\sigma$ of $60 \mathrm{Si} 2 \mathrm{Mn}$ is $1176 \mathrm{MPa}$, and the safety factor $\lambda$ is 1.5. Substituting the values of $\sigma_{s}$ and $\lambda$ into equation (13), we calculate that the material allowable stress $[\sigma]$ is $784 \mathrm{MPa}$. The maximum stress of the micromechanism is $30.964 \mathrm{MPa}$, which is far less than the allowable stress of the material.

According to the finite element analysis of statics, the maximum stress of the micro rotary mechanism during movement is far less than the allowable stress of the material, which indicates that the mechanism structure design is reasonable, and the mechanism satisfies the demand of the material strength check.

4.2. Modal Analysis. To determine whether the mechanism mechanism will cause resonance during motion and find the suitable work frequency range of the mechanism, it is necessary to analyze the inherent frequency of the micromechanism in its work environment. In this paper, the modal performance of the mechanism is analyzed by the dynamic modules of the FEM.

The modal analysis of the mechanism using the dynamic module of the FEM software, the modeling, and meshing of the mechanism are similar to the strength analysis (as in Section 4.1), and results as shown in Figure 6, because values of the first 6 orders are close to 0 , the $7^{\text {th }}-12^{\text {th }}$ orders can be regarded as the first 6 orders, and the first 6 orders of inherent frequency of the mechanism are shown in Figure 6.

The simulation results indicate that the micro-drive rotary system will cause no resonance during motion because the drive frequency of the PZT (drive frequency is $300 \mathrm{~Hz}$ ) does not coincide with each order of inherent frequency of the mechanism. 


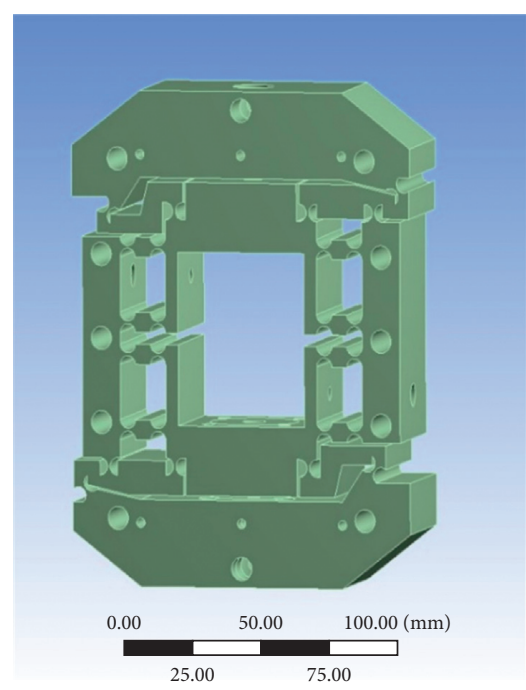

(a)

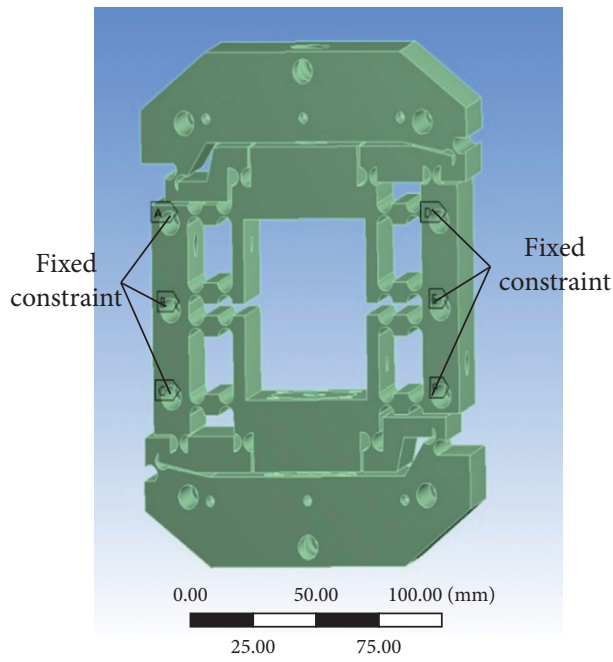

(c)

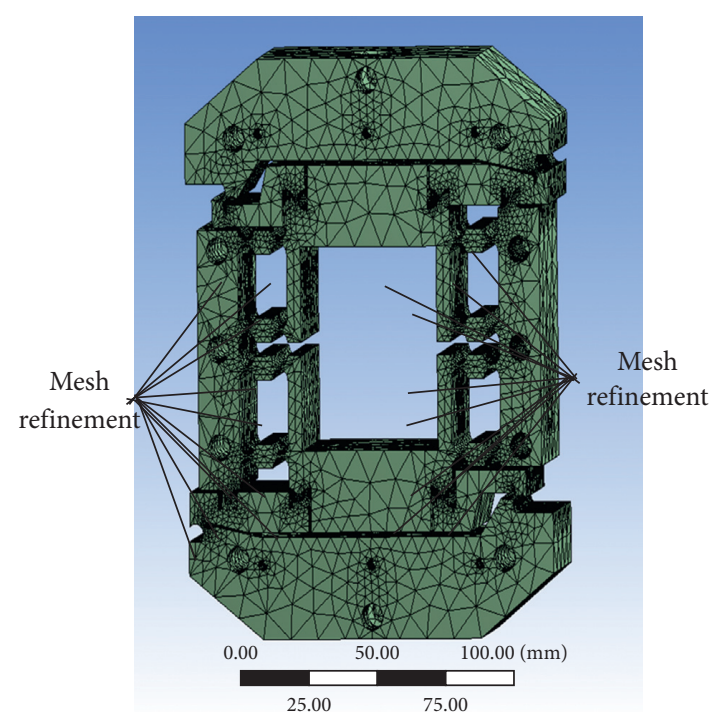

(b)

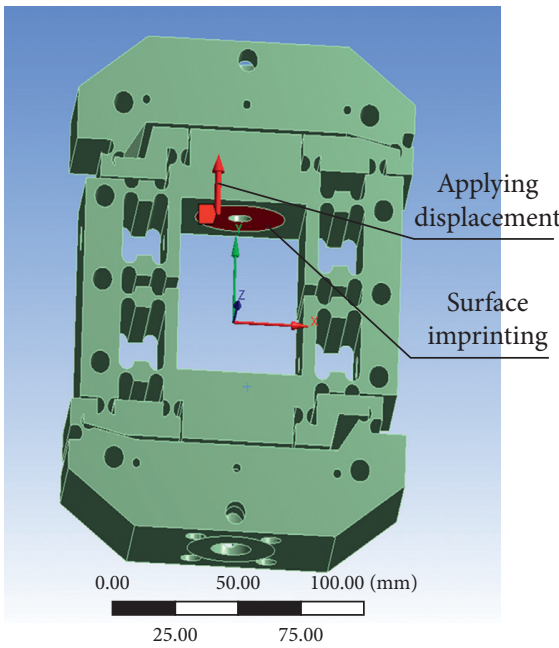

(d)

FIgURE 4: The preparation of FEM analysis. (a) Importing model. (b) Meshing. (c) Constraint condition. (d) Loading.

\section{Experimental Verification}

5.1. Modal Experiment. In the free dynamic experiment of the mechanism, the main experiment test apparatus is the dynamic test control analysis system produced by $M+P$ company, and other corollary equipment includes the data collection sensor, high-precision hammer of PCB, and elastic rope. To avoid the effect of the external environment, the dynamic experiment of the mechanism is finished using the free hanging method. In this experiment, the mechanism with free hanging is knocked by a high-precision hammer of $\mathrm{PCB}$, and the test data is recorded by the dynamic test control analysis system. The free dynamic experiment of the mechanism is performed as shown in Figure 7, and the test result of the experiment is shown in Figure 8.

The first six orders of inherent frequency of the mechanism are analyzed by the dynamic modules of the FEM software (as in Section 4.2) and free dynamic experiment results are shown in Figure 9.

The simulation and experiment results of the mechanism modal show two conclusions can be drawn as follows:

(1) The maximum error between the two analysis methods is $6.52 \%$, which shows that the simulation and experiment results are similar and accurate, and the system has excellent modal performance because the first-order inherent frequency of the mechanism is approximately $230 \mathrm{~Hz}$.

(2) The micro-drive system is driven by a PZT of model $\mathrm{P}-235.1 \mathrm{~S}$, which is produced by PI Company, and the drive frequency of the PZT is $300 \mathrm{~Hz}$. The second- and third-order inherent frequencies of the mechanism are $267.19 \mathrm{~Hz}$ and $925.00 \mathrm{~Hz}$. Therefore, the micro-drive rotary system will cause no resonance during motion. 


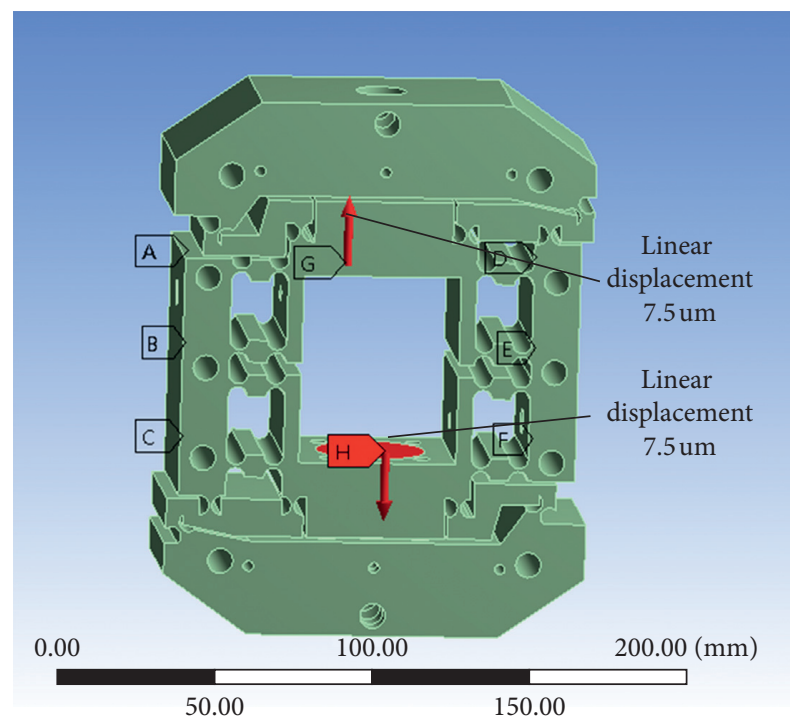

(a)
Maximum principal stress

Type: maximum principal stress

Unit: MPa

Time: 1

2015/6/15 21:15

\begin{tabular}{|l}
$30.964 \mathrm{Max}$ \\
27.299 \\
23.634 \\
19.968 \\
16.303 \\
12.638 \\
8.9723 \\
5.3069 \\
1.6416 \\
$-2.0238 \mathrm{Min}$
\end{tabular}

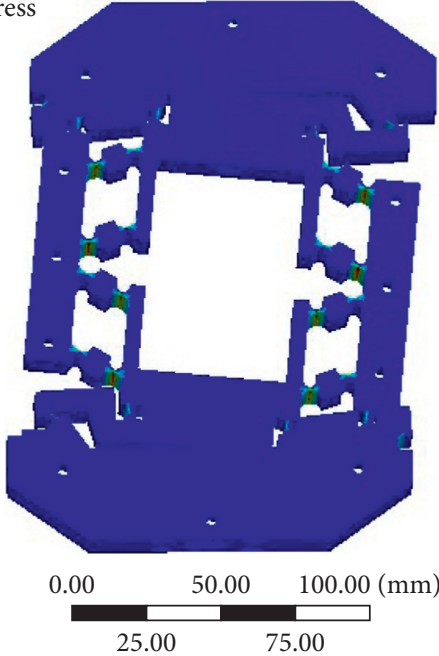

(b)

Figure 5: The maximum simulation stress of the micro rotary mechanism. (a) Loading displacements. (b) Result of simulation.

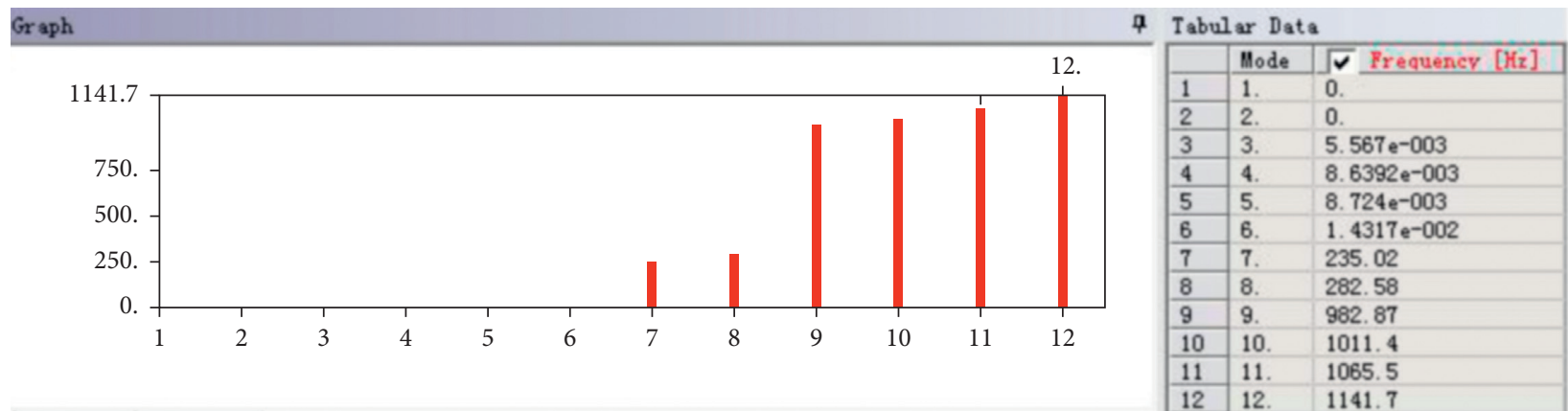

Figure 6: The first six-order $\left(7^{\text {th }}-12^{\text {th }}\right)$ inherent frequency of the mechanism.

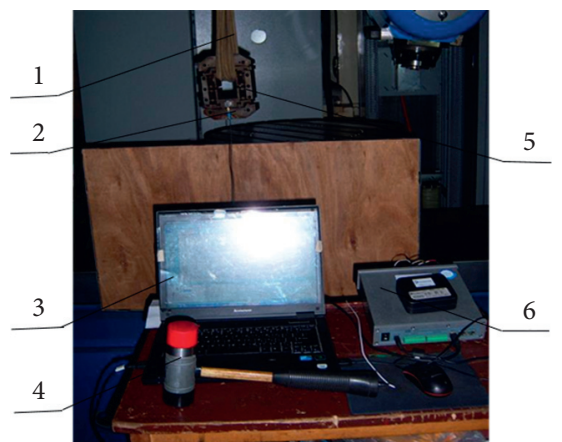

Figure 7: Free dynamic experiment of the mechanism. (1) Rubber rope. (2) Data acquisition front end. (3) Computer. (4) PCB high precision hammer. (5) Micro rotation mechanism. (6) The dynamic test control analysis system.

5.2. Error Compensation Performance Experiment. The micro-drive rotary system has different motion performance in the environment of the macro/micro dual-drive system and just micro-drive. To test the error compensation performance of the micro-drive rotary system, the experiment should be in the environment of the macro/micro dual-drive system. In this experiment, the function between driven voltage $U$ of the PZT and output motion $\Delta \theta$ of the 

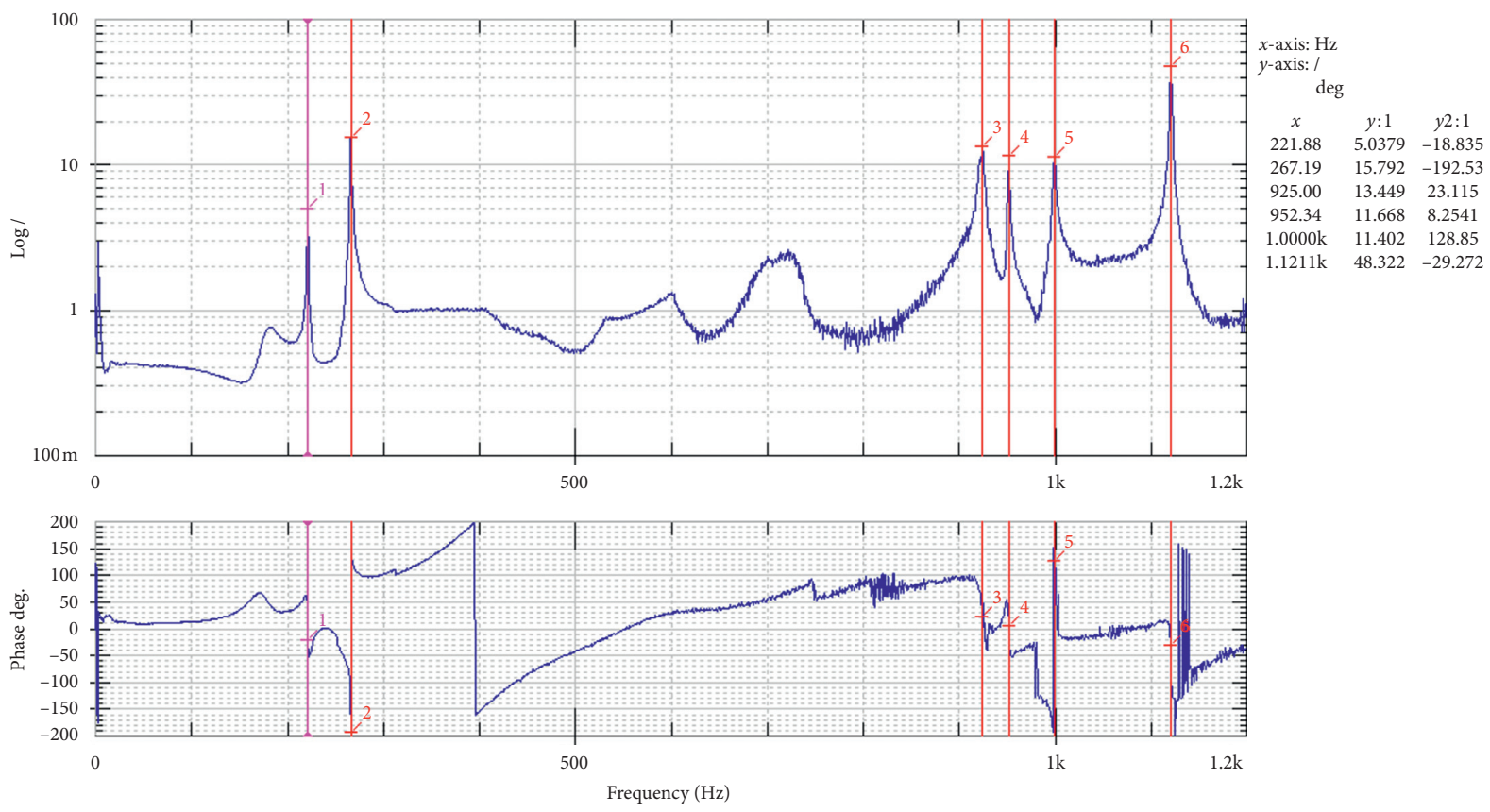

FIgURe 8: Result of the free dynamic experiment.

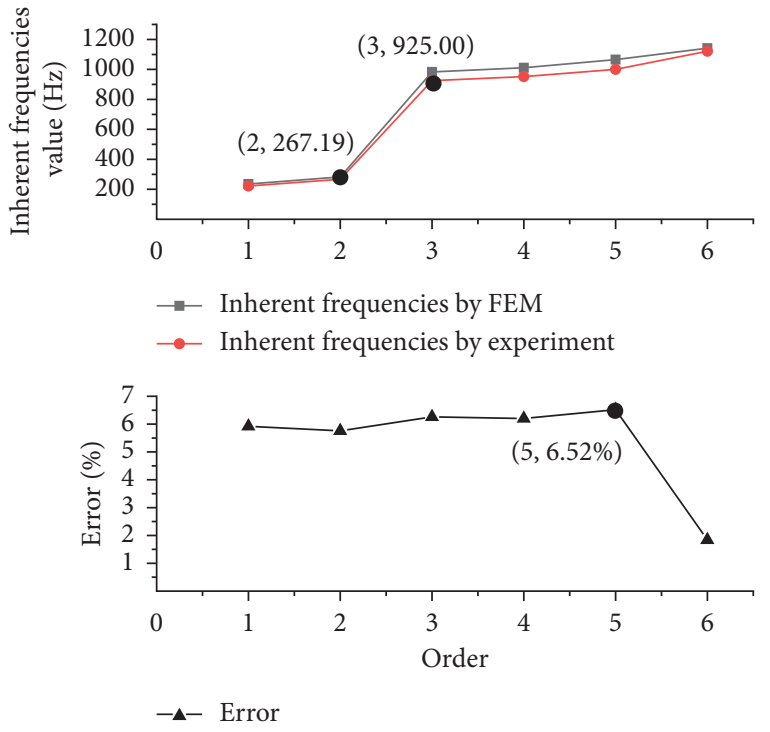

FIGURE 9: The simulation and experiment results of mechanism model.

micro-drive rotary system is analyzed, and driven voltage $U$ matching a known error compensation $\Delta \theta$ can be solved by the function.

To prevent external factors such as vibration and temperature variation from affecting the experiment results, the error compensation experiment is finished at a vibration isolation platform of the thermostatic laboratory.

In the error compensation experiment, the main experiment test apparatus includes the micro rotary mechanism, PZT, dual-frequency laser interferometer (model XL80 of Renishaw Company), control system of PMAC, direct drive motor (model DM1C-004 of Yokogawa Company), and experiment box. The error compensation experiment is shown in Figure 10. The temperature compensating sensor and humidity compensation sensor are installed on the two sides of the experiment box. In the environment of vibration isolation, constant temperature, and constant humidity, the output motion $\Delta \theta$ of the microdrive rotary system is detected by the dual-frequency laser interferometer when the system is driven with driven voltage $U$. The piezoelectric actuator is used for closed-loop feedback. Specifically, the precision displacement sensor inside the piezoelectric actuator is used for closed-loop feedback.

The range of driven voltage $U$ of the PZT is $0-10 \mathrm{~V}$ (because the PZT requires a small length to preload before working, and the maximum driven voltage $U$ is $9.8 \mathrm{~V}$ as 

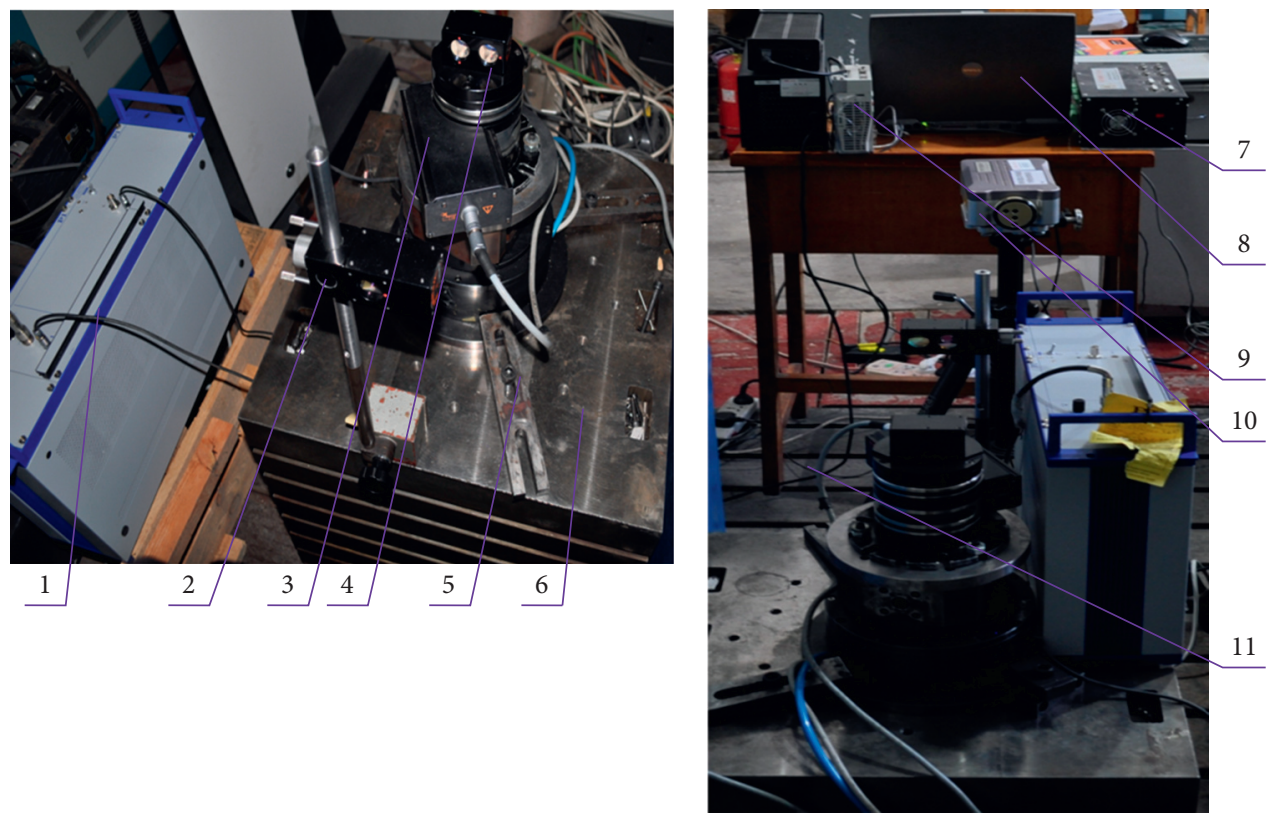

FIgURE 10: Compensation performance test diagram of the fretting rotary system. (1) PZT. (2) MirrorI. (3) Calibration shaft. (4) Mirror II. (5) Fixed clamp. (6) Experiment box. (7) Control system of PMAC. (8) Computer. (9) Diver of direct drive motor. (10) Dual-frequency laser interferometer. (11) Vibration isolation.

tested). To have a similar amount of elongation and shortening of PZT, the initial driven voltage $U$ of the PZT is set to $5 \mathrm{~V}$. The micro-drive rotary system can compensate the macro-drive rotary system with positive error and negative error. In the experiment, the PZT is controlled by closedloop control, the motor is stopping but under servo status to ensure motion in the environment of the macro/micro dualdrive system. The driven voltage $U$ of the PZT is set to $5 \mathrm{~V}$, and the current position of the calibration axis is set to the initial position (i.e., we define the position of the system with driven voltage $U$ of $5 \mathrm{~V}$ as the standard position). Then, we detect the output motion $\Delta \theta$ of the micro-drive rotary system in the ascent stage (driven voltage $U$ is $5-9.8 \mathrm{~V}$ ) and declining stage (driven voltage $U$ is $5-0 \mathrm{~V}$ ) using the dualfrequency laser interferometer, and the experiment results are shown in Figure 11.

To conveniently compensate for the errors of the microdrive rotary system, the motion of the system in the ascent stage and declining stage is linearly fitted according to the experiment result. The fitted equations are the equations of $U$ with error compensation. The linear-fitting of $U$ and $\Delta \theta$ is shown in Figure 12.

The linear fit equation of the micro-drive rotary system in the ascent stage is

$$
U=1.498 \Delta \theta+5.03 \text {. }
$$

The linearity of the linear equation is 0.9985 .

The linear fit equation of the micro-drive rotary system in the declining stage is

$$
U=1.596 \Delta \theta+4.82 \text {. }
$$

The linearity of the linear equation is 0.9952 .
When the micro-drive rotary system is compensating the errors of the macro-drive rotary system, if the required compensation angle $\Delta \theta$ is positive, the driven voltage $U$ can be calculated by equation (14) to be $5-9.8 \mathrm{~V}$. If the required compensation angle $\Delta \theta$ is negative, the driven voltage $U$ can be calculated by equation (15) to be $5-0 \mathrm{~V}$.

In order to facilitate the use of engineering, according to equations (14) and (15), if the initial driven voltage is a common value of $X(0 \leq X \leq 9.8)$, the driven voltage $(U)$ equation of error compensation is

$$
U= \begin{cases}1.498 \Delta \theta+X+0.03 & (0 \leq \Delta \theta \leq 6.1-0.62 X, \text { ascent stage }) \\ 1.596 \Delta \theta+X-0.18 & (-0.62 X \leq \Delta \theta \leq 0, \text { declining stage })\end{cases}
$$

In equation (16), the unit of voltage is volt (V), and the unit of error compensation angle $\Delta \theta$ is arc-second $\left({ }^{\prime \prime}\right)$. And when $0 \leq X \leq 9.8,-0.62 X \leq \Delta \theta \leq 6.1-0.62 X$.

Equation (16) is the driven voltage $(U)$ equation of error compensation when the initial driven voltage is $X$.

The error compensation performance experiment results show two conclusions can be drawn as follows:

(1) The driven voltage equation of error compensation is derived based on the experiment result, and the driven voltage $U$ can be calculated by equation (16) when $0 \leq X \leq 9.8$ ( $X$ is the initial driven voltage).

(2) The micro-drive rotary system can satisfy the error compensation demand of the macro-/micro dualdrive system, and the error compensation performance of the system is excellent. According to equation (16), the maximum error compensation angle $\left(\Delta \theta_{\max }\right)$ of the micro-drive rotary system is $6.1^{\prime \prime}$. In the macro/micro dual-drive system, the macro- 


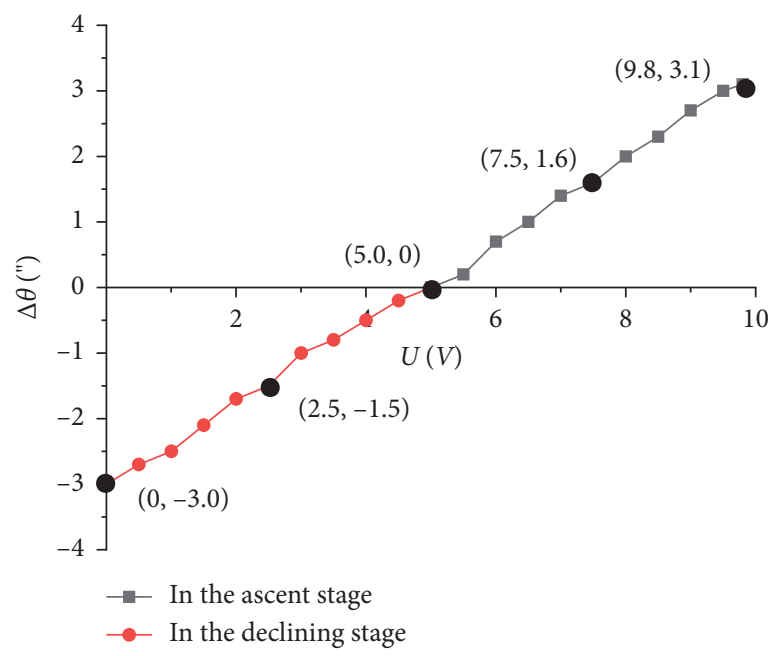

FIGURE 11: Error compensation performance experiment results.

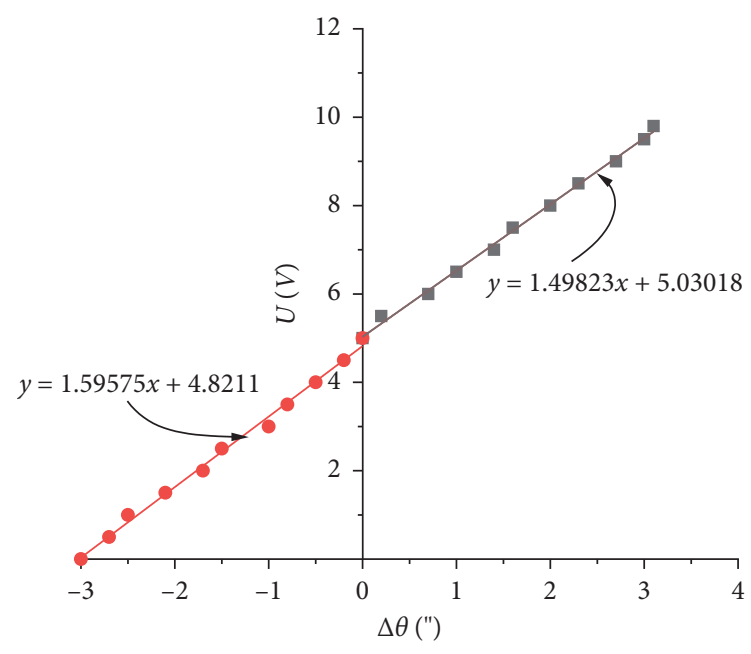

- In the ascent stage

- In the declining stage

The linear equation fitted in the ascent stage

- The linear equation fitted in the declining stage

FIgURE 12: The linear-fitting of $U$ and $\Delta \theta$.

drive system is driven by a direct drive motor of model DM1C-004, and the maximum positive and maximum negative errors of the motor are $2.0^{\prime \prime}$ and $-2.7^{\prime \prime}$. According to equations (5) and (12), the maximum compensation modifier $\left(\theta_{\max }^{\prime}\right)$ is $2.7^{\prime \prime}$. As $\Delta \theta_{\max }>\theta_{\max }^{\prime}$, the system can meet the error compensation demand in practical application.

\section{Conclusion}

In this paper, a new precision micro-drive rotary system is designed in order to obtain a high-precision rotary motion, the error compensation scheme of the system is proposed, and the system design and error compensation performances are verified by FEM simulation and experiments.

In the micro-drive rotary system, the micro rotary mechanism is driven by the PZT (the drive precision of PZT at $0.1 \mathrm{~nm}$ level). The system can accurately transform linear motion into rotary motion; therefore, the low-precision of micro rotary motion can be enhanced. According to simulation and experiments, the maximum stress of the mechanism is far less than the allowable stress $(30.964 \mathrm{MPa})$ of the material, and the micro-drive rotary system will cause no resonance during motion.

The error compensation equations (5) and (12) and the driven voltage equation of error compensation (16) are derived, and they are fit for engineering use. As the maximum error compensation angle $\left(\Delta \theta_{\max }\right)$ and the maximum compensation modifier $\left(\theta_{\max }^{\prime}\right)$ are $6.1^{\prime \prime}$ and $2.7^{\prime \prime}$, the microdrive rotary system can satisfy the error compensation demand of the macro/micro dual-drive system.

This study is beneficial to research on the micro-drive rotary system with high-precision (such as precision in subarc-second), and it is helpful to promote the development of the macro/micro dual-drive technology. In addition, the research results about error compensation of the system in this paper are useful for engineering practice.

\section{Data Availability}

The data used to support the findings of this study are available from the corresponding author upon request.

\section{Conflicts of Interest}

The authors declare no conflicts of interest with respect to the research in this article.

\section{Acknowledgments}

The authors would like to thank the support of the National Natural Science Foundation of China (51805428 and 51705417), the Basic Research Project of Natural Science in Shaanxi Province (2018JQ5205 and 2017JM5042), the Key Research and Development Project of Shaanxi Province (2016KTZDGY4-01), the Science and Technology Plan Project of Xi' an City (201805036YD14CG20 (7)), the Special 
Scientific Research Project of the Shaanxi Province Department of Education (18JK0528), and the Science and Technology Plan Project of Beilin District (GX2032).

\section{References}

[1] Y. W. Sun, M. S. Chen, J. J. Jia, Y. S. Lee, and D. M. Guo, "Jerklimited feedrate scheduling and optimization for five-axis machining using new piecewise linear programming approach," Science China Technological Sciences, vol. 62, no. 7, pp. 1067-1081, 2019.

[2] K. Ekholm, A. Moxnes, and K. H. Ulltveitmoe, "Manufacturing restructuring and the role of real exchange rate shocks," Journal of International Economics, vol. 86, pp. 101-117, 2012.

[3] F. C. Wang, L. S. Chen, Y. C. Tsai, C. H. Hsieh, and J. Y. Yen, "Robust loop-shaping control for a nano-positioning stage," Journal of Vibration and Control, vol. 20, no. 6, pp. 885-900, 2014.

[4] Y. C. Yang, X. Wang, J. W. Zhang et al., "Auto maticphaselocked control of grating tiling," Optics and Lasers in Engineering, vol. 50, no. 2, pp. 262-267, 2012.

[5] S. Y. Zeng and L. Blunt, "Experimental investigation and analytical modeling of the effects of process parameters on material removal rate for bonnet polishing of cobalt chrome alloy," Precision Engineering, vol. 38, no. 2, pp. 348-355, 2014.

[6] S. Zenla, L. B. Ayed, M. Nouari, and A. Delameziere, "Numerical prediction of the chip formation process and induced damage during the machining of carbon/epoxy composites," International Journal of Mechanical Sciences, vol. 90, pp. 89-101, 2015.

[7] H. J. Ahn, "Eddy current damper type reaction force compensation mechanism for linear motor motion stage," International Journal of Precision Engineering and Manufacturing-Green Technology, vol. 3, no. 1, pp. 67-74, 2016.

[8] E. L. Zheng, R. Zhu, S. H. Zhu, and X. J. Lu, "A study on dynamics of flexible multi-link mechanism including joints with clearance and lubrication for ultra-precision presses," Nonlinear Dynam, vol. 83, no. 1-2, pp. 137-159, 2016.

[9] S. L. Jiang and Y. W. Sun, "Stability analysis for a milling system considering multi-point-contact cross-axis mode coupling and cutter run-out effects," Mechanical Systems and Signal Processing, vol. 141, Article ID 106452, 2020.

[10] R. Majumdar and I. Paprotny, "Configurable post-release stress -engineering of surface micro-machined MEMS structures," Journal of Microelectromechanical Systems, vol. 26, no. 3, pp. 671-678, 2017.

[11] H. Itagaki and M. Tsutsumi, "Control system design of a linear motor feed drive system using virtual friction," Precision Engineering, vol. 38, no. 2, pp. 237-248, 2014.

[12] J. W. Wu, K. C. Huang, M. L. Chiang, M. Y. Chen, and L. C. Fu, "Modeling and controller design of a precision hybrid scanner for application in large measurement-range atomic force microscopy," IEEE of Thermal Power Electronics, vol. 61, no. 7, pp. 3704-3712, 2014.

[13] S. L. Xu, K. Shimada, M. Mizutani, and T. Kuriyagawa, "Fabrication of hybrid micro/nano-textured surfaces using rotary ultrasonic machining with one-point diamond tool," International Journal of Machine Tools and Manufacture, vol. 86, no. 6, pp. 12-17, 2014.

[14] D. Bassing and A. S. Braeurer, "The lag between micro-and macro-mixing in compressed fluid flows," Chemical Engineering and Sciences, vol. 163, pp. 105-113, 2017.
[15] L. Y. Zhang, J. Gao, X. Chen et al., "Implementation and experiment of an active vibration reduction strategy for macro-micro positioning system," Precision Engineering, vol. 51, pp. 319-330, 2018.

[16] C. You, M. H. Lee, H. J. Lee et al., "The effect of macro/micro combination pore structure of biphasic calcium phosphate scaffold on bioactivity," Ceramics International, vol. 43, no. 4, pp. 3540-3546, 2017.

[17] W. Tang, D. Lin, Y. M. Yu et al., "Bioinspired trimodal macro/ micro/nano-porous scaffolds loading rhBMP-2 for complete regeneration of critical size bone defect," Acta Biomateriallia, vol. 32, pp. 309-323, 2016.

[18] X. C. Zhuang, S. M. Ma, and Z. Zhao, "A microstructurebased macro-micro multi-scale fine-blanking simulation of ferrite-cementite steels," International Journal of Mechanical Sciences, vol. 128, pp. 14-427, 2017.

[19] Y. L. Yang, Y. D. Wei, J. Q. Lou, L. Fu, and X. W. Zhao, "Nonlinear dynamic analysis and optimal trajectory planning of a high-speed macro-micro manipulator," Journal of Sound and Vibration, vol. 405, pp. 112-132, 2017.

[20] X. Q. Sun and B. T. Yang, "A new methodology for developing flexure-hinged displacement amplifiers with micro-vibration suppression for a giant magnetostrictive micro-drive system," Sensors and Actuators A: Physical, vol. 263, pp. 30-43, 2017.

[21] M. Lok, E. F. Helbling, X. Zhang, R. Wood, D. Brooks, and G. Y. Wei, "A low mass power electronics unit to drive piezoelectric actuators for flying microrobots," IEEE of Thermal Power Electronics, vol. 33, no. 4, pp. 3180-3191, 2017.

[22] T. Mashimo, "Miniature preload mechanism for a micro ultrasonic motor," Sensors and Actuators A: Physical, vol. 257, pp. 106-112, 2017.

[23] E. Hosseinian, P. O. Theillet, and O. N. Pierron, "Temperature and humidity effects on the quality factor of a silicon lateral rotary micro-resonator in atmospheric air," Sensors and Actuators A: Physical, vol. 189, pp. 380-389, 2013.

[24] D. C. Zhu and Y. P. Feng, "Structure design of a 3-DOF UPC type rotational fully spatial compliant parallel manipulator," International Journal of Advancements in Computing Technology, vol. 5, no. 8, pp. 70-81, 2013.

[25] R. Pan, X. J. Zhao, P. T. Liu, and R. M. Ren, "Micro-mechanism of polygonization wear on railroad wheels," Wear, vol. 392, pp. 213-220, 2017.

[26] X. J. Fan, M. M. Sun, Z. H. Lin et al., "Automated noncontact micromanipulation using magnetic swimming microrobots," IEEE of Thermo Nanotechnology, vol. 90, 2018.

[27] M. A. A. Fenelon and T. Furukawa, "Design of an active flapping wing mechanism and a micro aerial vehicle using a rotary actuator," Mechanism and Machine Theory, vol. 45, no. 2, pp. 137-146, 2010.

[28] D. M. Brouwer, BR. D. Jong, and H. M. J. R. Soemersa, "Design and modeling of a six DOFs MEMS-based precision manipulator," Precision Engineering, vol. 34, no. 2, pp. 307319, 2010.

[29] K. Kim, D. Ahn, and D. Gweon, "Optimal design of a 1rotational DOF flexure joint for a 3 DOF H-type stage," Mechatronics, vol. 22, pp. 24-32, 2011.

[30] J. H. Qu, J. Tang, Y. B. Gianchandani, K. R. Oldham, and S. R. Green, "Dynamic modeling of a bidirectional magnetoelastic rotary micro-motor," Sensors and Actuators A: Physical, vol. 223, pp. 49-60, 2015.

[31] G. P. Xue, M. Toda, and T. Ono, "Comb-drive XYZ-microstage with large displacements based on chip-level microassembly," Journal of Microelectromechanical and Sciences, vol. 25, no. 6, pp. 989-998, 2016. 
[32] M. Liu, X. M. Zhang, and S. Fatikow, "Design and analysis of a multi-notched flexure hinge for compliant mechanisms," Precision Engineering, vol. 48, pp. 92-304, 2017.

[33] D. F. Machekposhti, N. Tolou, and J. L. Herder, "A statically balanced fully compliant power transmission mechanism between parallel rotational axes," Mechanism \& Machine Theory, vol. 119, pp. 51-60, 2018.

[34] C. Du, Y. Zhang, A. Kong, and Z. Yuan, "High-precision and fast response control for complex mechanical systems-servo performance of dedicated servo recording systems," IEEE Transactions on Magnetics, vol. 53, no. 3, pp. 1-5, 2017.

[35] Z. X. Shao, S. L. Wu, J. G. Wu, and H. Y. Fu, "A novel 5-DOF high-precision compliant parallel mechanism for large-aperture grating tiling," Mechical Sciences, vol. 8, no. 2, pp. 349-358, 2017.

[36] F. Jiang, J. Chen, H. Bi, L. Y. Li, W. K. Jing, and J. Zhang, "The underlying micro-mechanism of performance enhancement of non-polar $n-\mathrm{ZnO} / p$-AlGaN ultraviolet light emitting diode with $i$-ZnO inserted layer," Applied Physics Letters, vol. 112, no. 3, Article ID 033505, 2018.

[37] Y. X. Peng, S. Ito, Y. Shimizu, and W. Gao, "A micro-stage for linear-rotary positioning," Key Engineering Materials, vol. 523, pp. 650-655, 2012.

[38] W. X. Xu and Y. B. Wu, "Piezoelectric actuator for machining on macro-to-micro cylindrical components by a precision rotary motion control," Mechanical Systems and Signal Processing, vol. 114, pp. 439-447, 2019. 